\title{
Nonalcoholic fatty liver disease and atrial fibrillation: possible pathophysiological links and therapeutic interventions
}

\author{
Hossein Haghbina, Manesh Kumar Gangwani ${ }^{b}$, Shri Jai Kirshan Ravic, Abhilash Perisettid, \\ Muhammad Aziza, Hemant Goyale, Ali Nawrasf, Thomas Sodeman ${ }^{f}$
}

University of Toledo Medical Center, Toledo, Ohio; Mercy Hospital St. Louis, Missouri; Guthrie Robert Packer Hospital, Pennsylvania; University of Arkansas for Medical Sciences, Arkansas; The Wright Center for Graduate Medical Education, Pennsylvania, USA

\section{Abstract}

\begin{abstract}
Atrial fibrillation (AF) and nonalcoholic fatty liver disease (NAFLD) share common risk factors and appear to have an association. Independently, the incidence and prevalence of both diseases are on the rise. Epidemiological evidence, experimental studies and various randomized clinical trials suggest a link between the 2 entities, delineating cumulative risks and clinical strategies to improve outcomes. Dyslipidemia, insulin resistance, inflammatory milieu, and activation of the reninangiotensin system are likely common pathophysiological mechanisms linking AF and NAFLD. In this article we review the known pathways and pathophysiology that link the 2 conditions. This review also discusses therapies that target both NAFLD and AF, such as angiotensin-converting enzyme inhibitors/angiotensin receptor blockers, statins, metformin, and vitamin E. We further discuss other potential medications that have shown effects in NAFLD or AF through anti-inflammatory, antidiabetic, lipid-lowering, or renin-angiotensin system inhibiting effects. Future epidemiological studies are needed to establish a direct causal relationship between NAFLD and AF.
\end{abstract}

Keywords Non-alcoholic fatty liver disease, atrial fibrillation, treatment, intervention, pathophysiology

Ann Gastroenterol 2020; 33 (6): 1-12

\section{Introduction}

Nonalcoholic fatty liver disease (NAFLD) is defined as the imaging or histologic evidence of an accumulation of triglycerides in hepatocytes in the absence of secondary causes, such as excessive alcohol intake, viral hepatitis, medications or other cause of liver injury. NAFLD includes

aDepartment of Internal Medicine, University of Toledo Medical Center, Toledo, Ohio (Hossein Haghbin, Muhammad Aziz);

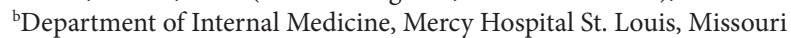
(Manesh Kumar Gangwani); 'Department of Internal Medicine, Guthrie Robert Packer Hospital, Pennsylvania (Shri Jai Kirshan Ravi); ${ }^{\mathrm{D} D e p a r t m e n t}$ of Gastroenterology and Hepatology, University of Arkansas for Medical Sciences, Arkansas (Abhilash Perisetti); ${ }^{e}$ Division of Gastroenterology, The Wright Center for Graduate Medical Education, Pennsylvania (Hemant Goyal); fDivision of Gastroenterology and Hepatology, University of Toledo Medical Center, Toledo, Ohio (Ali Nawras, Thomas Sodeman), USA

\section{Conflict of Interest: None}

Correspondence to: Muhammad Aziz, MD, University of Toledo Medical Center, Toledo, Ohio, United States, e-mail: marajani@hotmail.com

Received 3 May 2020; accepted 4 August 2020;

published online 12 October 2020

DOI: https://doi.org/10.20524/aog.2020.0550 a spectrum of disease severity ranging from simple steatosis with minimal liver injury to hepatitis, defined as nonalcoholic steatohepatitis (NASH), with increasing levels of liver fibrosis eventually leading to cirrhosis [1]. The main risk factors for NAFLD are concomitant metabolic syndrome, type 2 diabetes mellitus (DM-2) and obesity. Both NAFLD and its risk factors are prevalent in the western world, where NAFLD is the most common cause of liver disease [2]. According to the Third National Health and Nutrition Examination Survey, roughly $10-40 \%$ of Americans have NAFLD [3]. Another concerning trend is that more adolescents are being diagnosed with NAFLD, with a prevalence of 3-18\% [4]. As NAFLD is a progressive disease that leads to cirrhosis and adds to overall mortality, adolescents will experience significant cumulative morbidity and mortality in their lifetime [5].

Atrial fibrillation $(\mathrm{AF})$ is the most common cardiac arrhythmia in the elderly, coexisting with other comorbidities [6]. The prevalence of AF in the United States (US) was about 3 million in 2005 and is projected to increase to 7.5 million by 2050 [7]. Multiple risk factors, including hypertension, advanced age, obesity, DM-2, smoking and other components of metabolic syndrome (such as central obesity, dyslipidemia and insulin resistance), are contributing to an increasing incidence of $\mathrm{AF}$ [8]. In their review, Wasmer et al noted an increase in AF incidence in the younger population and proposed lifestyle changes leading 
to obesity as the most likely cause [9]. Binge drinking, leading to "holiday heart syndrome", is another possible mechanism of increased AF in the young population [10]. However, none of these factors are a well-established cause of the early onset of AF. Therefore, it is essential to investigate a causal or bidirectional relationship to better understand the pathophysiological relationship between $\mathrm{AF}$ and other disease states.

In recent years, a large amount of evidence has emerged to support the association between NAFLD and AF. Multiple mechanisms have been described, but the pathophysiology is not completely understood. Previous review articles, including a meta-analysis, have attempted to establish a relation between
NAFLD and AF. We tried to build on these review articles and further provide a section on pharmacological interventions, including experimental drugs, that might offer a potential therapeutic option that targets both entities $[11,12]$.

\section{NAFLD and AF: epidemiological coexistence}

There are multiple hypotheses to explain the correlation between NAFLD and AF (Fig. 1). Various studies have delineated the correlation as a primary or secondary finding.

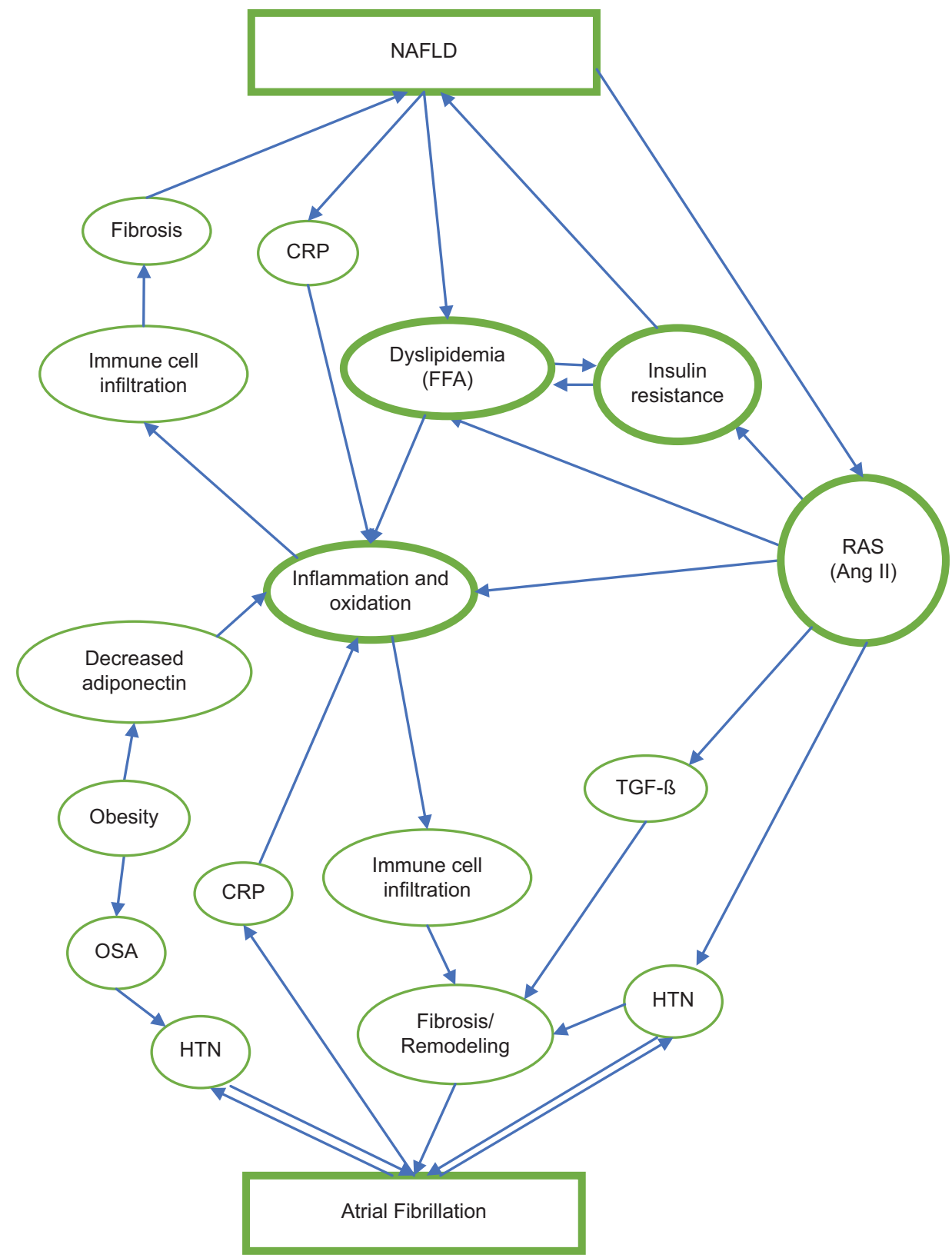

Figure 1 Pathophysiology linking NAFLD and atrial fibrillation

Ang II, angiotensin II; CRP, C-reactive protein; FFA, free fatty acid; HTN, hypertension; NAFLD, nonalcoholic fatty liver disease; OSA, obstructive sleep apnea; RAS, renin-angiotensin system; TGF- $\beta$, transforming growth factor- $\beta$ 
The most likely explanation is the presence of common risk factors shared between the two conditions.

In 2013, Framingham Heart Study researchers evaluated 3744 AF patients over 10 years. After adjustment for risk factors such as age, body mass index (BMI), DM-2, etc., the researchers found that elevated levels of serum transaminases (regardless of the cause of liver disease) were independent risk factors for AF. This was still the case after the exclusion of patients with moderate to severe alcohol intake [13]. Although the Framingham study did not specifically investigate NAFLD, Clark et al illustrated that the etiology of transaminase in the US is NAFLD [14]; therefore, although weak, there is evidence that AF maybe associated with NAFLD.

Zhang et al studied 1688 Chinese adults to determine the association between AF and NAFLD. NAFLD diagnosis was based on sonographic criteria. Most of the associative factors, including age, sex, BMI, systolic and diastolic blood pressures, and lipid profile, were adjusted for as confounders and the results showed a statistically significant correlation between NAFLD and AF (odds ratio [OR] 1.95, 95\% confidence interval [CI] 1.03-3.69) [15]. You et al performed the largest longitudinal study to date on 232,979 adult Korean subjects from 2009 to 2013 , with a mean follow-up period of 3.7 years. After adjusting for confounders such as age, sex, heart failure, chronic kidney disease, obesity, impaired fasting glucose and systolic blood pressure, the hazard ratio (HR) for AF associated with NAFLD was 1.13 (95\%CI 1.03-1.24; $\mathrm{P}=0.01$ ). Moreover, for every 10 -unit increase in the fatty liver index (a score based on anthropometric and laboratory values for prediction of the fatty liver), the risk of AF increased by 4\% [16]. Karajmaki et al performed a prospective study on 958 middle-aged Finnish individuals from the OPERA (Oulu Project Elucidating Risk of Atherosclerosis) cohort with a 16-year follow-up period. The researchers found a 2-fold greater incidence of AF in NAFLD patients $(14.9 \%$ vs. $7.9 \%, \mathrm{P}<0.001)$. NAFLD was a risk factor for AF after adjustment for confounders, including age, sex, diabetes, BMI, serum alanine aminotransferase, systolic blood pressure, left ventricular mass index, left atrial diameter, coronary artery disease and C-reactive protein (CRP) (adjusted OR 1.88, 95\%CI 1.03-3.45) [17]. The same group studied the gradient correlation of liver stiffness (a surrogate of liver fibrosis) with AF using transient elastography (TE) and echocardiography. In a crosssectional study, they divided 76 subjects from the OPERA study into 4 subgroups with and without NAFLD or AF. Left atrial diameter was used as a surrogate of AF severity. They concluded that patients with AF had higher TE values, with a proportional increment relation between TE value and left atrial diameter (by TE tertiles: 39, 45 and $48 \mathrm{~mm}, \mathrm{P}<0.001$ ) [18]. The results of this study suggest a gradient relationship between NAFLD and AF.

Though most studies have consistently shown a positive relationship between NAFLD and AF, some have presented negative results. Mantovani et al conducted a meta-analysis on 364,919 patients from 5 cross-sectional and 4 longitudinal studies. Their analysis of the 5 cross-sectional studies found NAFLD to be increasingly associated with the prevalence of $\mathrm{AF}$, after adjustment for confounders such as age, sex, BMI and hypertension (HTN) (adjusted OR 2.07, 95\%CI 1.38-3.10;
$\left.I^{2}=54.7 \%\right)$. However, the analysis of 4 longitudinal studies (including You et al and Karajmaki et al) showed NAFLD as a predictor for 10-year increased risk of incident AF only in DM-2 patients (HR 4.96, 95\%CI 1.42-17.28) [12].

In order to search for a missing link between $\mathrm{AF}$ and NAFLD other than metabolic syndrome (including HTN, DM2, or obesity), Mahfouz et al hypothesized that interatrial septal thickness (IAST) and left atrial stiffness (LASt) cause AF in patients with NAFLD. They performed an echocardiography and speckletracking assessment of left atrial function to determine IAST and LASt in 180 patients with NAFLD. The study findings suggested that high values of IAST and LASt were associated with a greater incidence of AF in NAFLD. IAST and LASt are independent risk factors and can serve as a mechanistic link between the 2 diseases [19]. The authors hypothesized that myocardial steatosis might exert local adverse effects leading to cardiac autonomic changes, as well as structural and electrical remodeling, causing the heart to become pro-arrhythmogenic. However, we believe the link that Mahfouz et al hypothesized was questionable. Although the authors performed multivariate regression analysis to determine the independence of IAST and LASt from confounding factors, there are proinflammatory cytokines from NAFLD livers that may promote AF [20]. Other investigators have also found LASt to be an independent risk factor for AF; however, they did not control for NAFLD or for proinflammatory cytokines as confounding factors [21]. Another link between AF and obesity is pericardial fat. It has been shown that there is a gradient relationship between the two, and that therapies that reduce pericardial fat can reduce the risk of AF. The proposed pathology is that pericardial fat tissue is a source of proinflammatory cytokines that exert local arrhythmogenic effects and cause AF [22].

An important limitation to consider is the fact that some studies did not take into account possible confounders. Future studies should be conducted to assess the role of confounders in the pathophysiological link between AF and NAFLD.

\section{Putative mechanisms}

One mechanism linking AF and NAFLD in obese individuals is the inflammatory and oxidative system. Inflammation and oxidative stress are closely connected, as the innate arm of the immune system produces reactive oxidation species via the myeloperoxidase enzyme [23]. Obesity leads to decreased release of an anti-inflammatory mediator called adiponectin and increased release of inflammatory mediators such as interleukin (IL)-6, tumor necrosis factor (TNF)- $\alpha$, and CRP from adipose tissue, leading to a proinflammatory milieu and oxidative stress [24]. Cell death and the ensuing fibrosis from oxidative radicals and inflammatory infiltration lead to the progression of both AF and NAFLD [25]. Cardiac myocytes show both cellular (modification of ion currents) and structural (fibrosis) alterations, leading to AF [26]. For example, a case-control study by Ozveren et al showed a longer interatrial electromechanical delay and conduction abnormality in NAFLD patients, after adjustment for HTN and heart disease [27]. This is due to both increased fibrosis and the cellular ion channelopathies observed 
in the cardiomyocytes of NAFLD patients. To close the causation loop, a liver with NAFLD produces atherogenic lipids and inflammatory proteins like CRP, leading to a proinflammatory and pro-oxidant milieu [28,29].

The renin-angiotensin system (RAS) is activated in patients with NAFLD [30]. The RAS contributes to HTN and can also lead to heart fibrosis. Angiotensin II is a proinflammatory component that causes an increase in cytokines, such as IL-6 and TNF- $\alpha$, that worsen NAFLD through hepatic infiltration of inflammatory cells [31]. Angiotensin II leads to fatty accumulation in the liver via an altered metabolism of fatty acids and very-lowdensity lipoproteins [32]. In addition, angiotensin II promotes oxidative stress and leads to insulin resistance [33]. Angiotensin II leads to transforming growth factor (TGF)- $\beta$-mediated cardiac fibrosis, resulting in left atrial electrical and mechanical remodeling that can lead to AF [34]. Interestingly, although evidence is limited, $\mathrm{AF}$ can itself further produce an inflammatory state and sustain it, rendering the relationship bidirectional. Evidence of AF-mediated inflammation is that cardioversion can lead to a decrease in CRP level; the proposed mechanism is myocyte calcium overload in the heart with AF and cell death [35].

\section{Potential therapies targeting NAFLD and AF}

\section{Lifestyle interventions}

No studies have investigated the effects of lifestyle on AF and NAFLD concurrently. A weight loss of 5-7\% through lifestyle modification with diet and exercise is the mainstay of the management of overweight patients with NAFLD and the only treatment for fibrosis regression [36]. Regarding AF, the LEGACY and REVERSE-AF studies have shown a decrease in the progression of AF, or even reversal, after weight loss [37,38]. Obesity leads to inflammatory mediator release from adipose tissue, increasing AF and NASH [24,25]. Khanna et al showed that a low-calorie diet and exercise-induced weight loss led to a decrease in inflammatory markers (IL-6 and TNF- $\alpha$ ) [39]. A meta-analysis of 116 studies by Askarpour et al found significant improvement in CRP $(\mathrm{P}<0.001)$, IL-6 $(\mathrm{P}<0.001)$, and TNF- $\alpha(\mathrm{P}=0.031)$ in obese patients after bariatric surgery [40]. Further studies have shown that exercise leads to improvement in lipid profile, glycemic index and blood pressure, and a decrease in systemic inflammatory markers (CRP) [41]. Colbert et al, in a study involving over 3000 subjects, found evidence that exercise causes a decrease in IL-6 and CRP $(\mathrm{P}<0.05)$. Interestingly, the effect was still present even after adjustment for weight loss; thus, exercise exerts its effects through other mechanisms than just weight loss alone [42].

\section{Pharmacological interventions}

Unfortunately, many patients find it challenging to institute lifestyle changes over the long term [43]. Many medications that act on AF are also seen to be effective for NAFLD, and vice versa, because of the suggested common pathophysiological pathway (Table 1 and Fig. 2). Medications effective for the conditions target the 4 pathways: namely RAS, hyperglycemia, dyslipidemia, and inflammation. The medications with a proven effect on both conditions are angiotensin-converting enzyme inhibitors (ACEI) and angiotensin receptor blockers (ARBs) (only animal studies), pioglitazone, dipeptidyl peptidase-4 (DPP-4) inhibitor, glucagon-like peptide-1 (GLP-1) analogs, statins, and vitamin $\mathrm{E}$. In addition to the well-established medications, we highlight a list of developing/novel therapies for NAFLD that have potential to be effective for AF (Table 1). Following are the established and proposed experimental medications categorized based on their mechanism of action. Note that some of the medications are known to have multiple mechanisms. Unfortunately, we were unable to account for baseline therapies for AF, such as anticoagulants and/or antiarrhythmics, as they can be a potential confounder in treatment for AF and NAFLD.

\section{Antidiabetic medications}

\section{Metformin}

Metformin has been extensively studied in NAFLD. In a meta-analysis of 9 randomized controlled trials (RCTs) and 417 participants by $\mathrm{Li}$ et al, it was shown that metformin has beneficial metabolic effects but no histological effects in NAFLD [44]. In a nationwide study of a cohort of 645,710 patients, Chang et al showed that metformin decreases the chance of AF [45]. Metformin decreases hepatic gluconeogenesis and is the primary initial medication in diabetes mellitus [46]. Metformin has been shown to exert anti-inflammatory and antioxidant effects after adjustment for glycemic results. Metformin lowers serum CRP levels and increases nitric oxide, leading to anti-inflammatory effects [47].

\section{Pioglitazone}

In a meta-analysis of 4 RCTs from 3 continents, pioglitazone, a thiazolidinedione, was shown to significantly improve fibrosis in the liver of NASH patients compared to controls (OR 1.7, 95\%CI 1.0-2.8) [48]. A meta-analysis by Zhang et al of 3 RCTs and 4 observational studies including 130,854 patients showed that thiazolidinedione-treated patients have a $30 \%$ lower risk of developing AF compared to controls (OR 0.73, 95\%CI 0.62-0.87) [49]. In an RCT that included 23 DM-2 patients, pioglitazone was shown to reduce insulin resistance, free fatty acids and TNF- $\alpha$, which translates to an improved inflammatory and hypercoagulable environment [50].

\section{DPP-4 inhibitors and GLP-1 analogs}

DDP-4 inhibitors and GLP-1 are medications for diabetes that exert their effect via the activation of GLP-1 receptors. GLP-1 has been shown to have protective effects on cardiomyocyte contractility, glucose uptake and survival [51]. Clinically, GLP-1 has been shown to improve patients' ejection fraction and functional status [52]. Liraglutide (a GLP-1 
Table 1 Medications with effects on non-alcoholic fatty liver disease and atrial fibrillation

\begin{tabular}{|c|c|c|c|}
\hline Drug & NAFLD effects & AF effects & Mechanism of action \\
\hline $\mathrm{ACEI} / \mathrm{ARB}^{*}$ & $+^{\mathrm{a}}$ & + & RAS and TGF- $\beta$ inhibition \\
\hline Ezetimibe & + & $+/ 0$ & Intestinal cholesterol absorption inhibition \\
\hline DPP-4 inhibitor and GLP-1 analogs* & + & $t^{\mathrm{a}}$ & Insulin release and cardio-protection \\
\hline Metformin & $+/ 0$ & + & Decrease gluconeogenesis \\
\hline Obeticholic acid & + & 0 & FXR agonist \\
\hline Orlistat & + & 0 & Intestinal lipase inhibitor \\
\hline Pentoxifylline & + & $+/ 0$ & Phosphodiesterase inhibitor \\
\hline Pioglitazone ${ }^{*}$ & + & + & Stimulates PPAR- $\gamma$ \\
\hline Propranolol & $+^{\mathrm{a}}$ & + & $\beta_{1}$ and $\beta_{2}$ inhibition \\
\hline Statins ${ }^{*}$ & + & + & Inhibit HMG-CoA reductase and TGF- $\beta$ \\
\hline Silymarin & + & 0 & Antioxidant and anti-inflammatory \\
\hline Ursodeoxycholic acid & + & $+/ 0$ & Stimulates excretion of bile acids \\
\hline Vitamin $\mathrm{E}^{*}$ & + & + & Anti-inflammatory activity \\
\hline \multicolumn{4}{|l|}{ Experimental medications } \\
\hline Aramchol & + & 0 & Lipid synthesis inhibitor \\
\hline Belapectin & + & 0 & Galectin-3 inhibitor \\
\hline DUR-928 & $t^{\mathrm{a}}$ & 0 & LXR inhibitor \\
\hline Elafibranor & + & 0 & Agonist of PPAR- $\alpha$ and PPAR- $\delta$ \\
\hline Pradigastat & + & 0 & DGAT-1 inhibitor \\
\hline Quercetin & $t^{\mathrm{a}}$ & 0 & Antioxidant \\
\hline Tropifexor/Cenicriviroc & $t^{\mathrm{a}}$ & 0 & FXR agonist/ CCR2 and 5 inhibitor \\
\hline
\end{tabular}

agonist) has been proven to reduce AF inducibility in animal models $(\mathrm{P}=0.02)$ [53]. The SCALE trial showed that liraglutide also causes significant weight loss in obese patients [54] and reduces obstructive sleep apnea [55], 2 major risk factors for AF. Liraglutide has been shown to downregulate TNF- $\alpha$-induced oxidative stress [56] and endothelial inflammation [57]. The Liraglutide Efficacy and Action in NASH (LEAN) trial, which included 52 patients with NASH, showed a reduction in fibrosis progression from $36 \%$ in controls to $9 \%$ in the liraglutide group (relative risk 0.2, 95\%CI 0.1-1.0) [58].

\section{Anti-inflammatory and antioxidants}

\section{Pentoxifylline}

Pentoxifylline, a synthetic methylxanthine, is a phosphodiesterase inhibitor with cardiovascular protective effects achieved through smooth muscle relaxation, a decrease in neutrophil superoxide production, migration, and response to TNF- $\alpha$ and IL-1 [59]. A meta-analysis of 3 RCTs and 2 cohort studies showed that pentoxifylline treatment results in weight loss, improved transaminases and improved histological parameters in patients with NASH [60].

\section{Vitamin $\mathrm{E}$}

The American Association for the Study of Liver Diseases recommends vitamin $\mathrm{E}$ in patients with NASH [61]. Vitamin E decreases the levels pf proinflammatory cytokines like IL-8 and CRP via protein kinase $\mathrm{C}$ and cyclooxygenase-2 pathways [62]. An RCT by Rodrigo et al, which included 152 cardiac surgery patients, showed a reduction of postoperative AF in cardiac surgery patients on vitamin E [63]. However, vitamin E has not been established as a standard of care for AF patients.

\section{RAS inhibitors and sympatholytics}

\section{ACEl and ARBs}

ACEIs and ARBs are groups of medications with direct effect on RAS. Treatment with ACEIs and ARBs leads to 


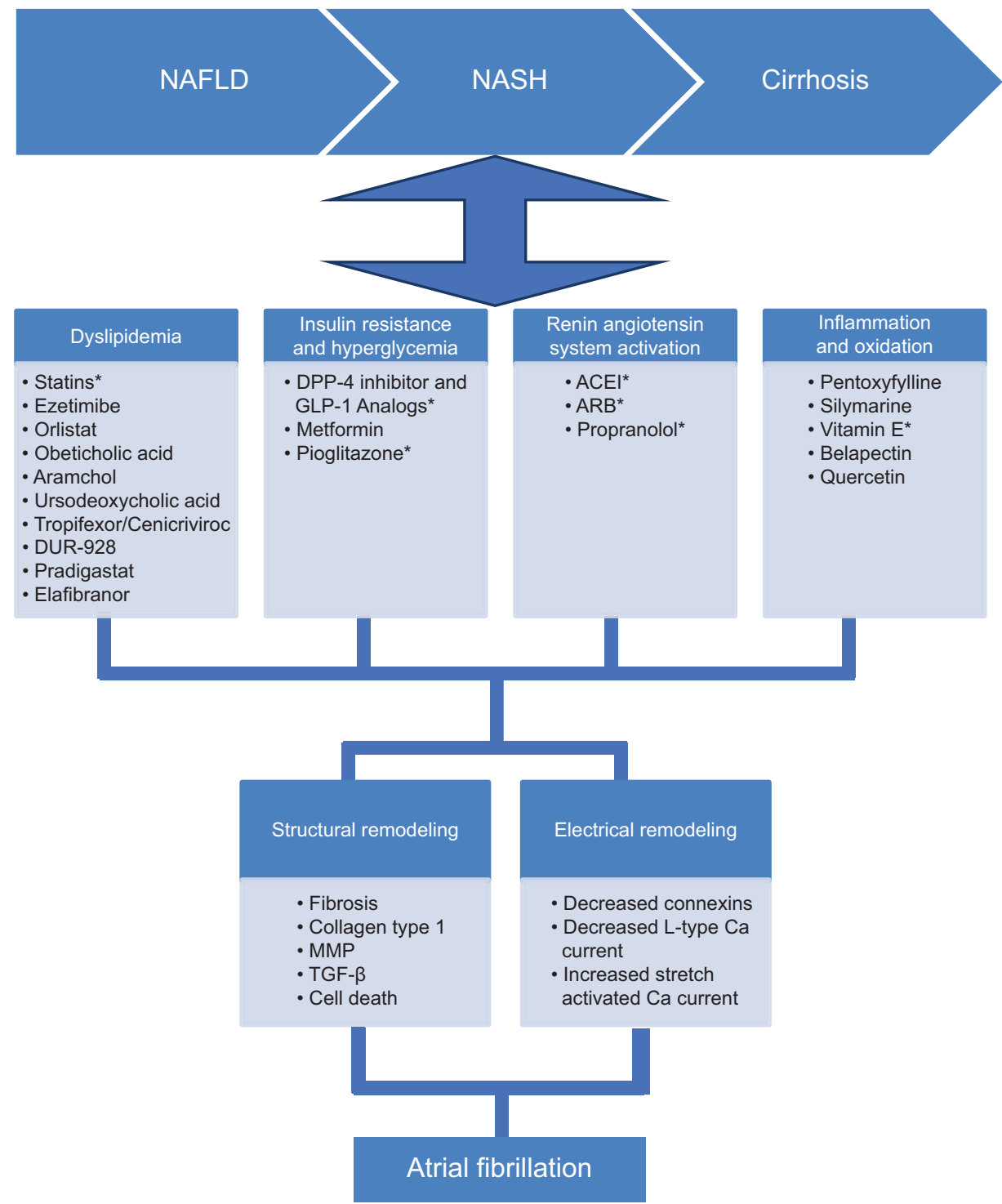

Figure 2 Drug therapy effects on pathways linking NAFLD and atrial fibrillation

MMP, matrix metalloproteinase; TGF- $\beta$, transforming growth factor- $\beta$; NAFLD, nonalcoholic fatty liver disease; NASH, nonalcoholic steatohepatitis; DPP-4, dipeptidyl peptidase-4; GLP-1, glucagon-like peptide-1; ACEI, angiotensin-converting enzyme inhibitor; ARB, angiotensin receptor blocker

attenuation of the RAS and beneficial effects on both NAFLD and AF in animal models [64]. There are other mechanisms apart from attenuating the RAS. Losartn, an ARB, inhibits Toll-like receptor- 4 and TGF- $\beta$ and reduces liver fibrosis in rats [65]. In animal models, valsartan ameliorates liver fibrosis by reducing TGF- $\beta$ and TNF- $\alpha$, in addition to its antiapoptotic effects [66]. ACEI and ARBs also lead to a decrease in AF via a decrease in cardiac fibrosis, in addition to the other mechanisms [67].

\section{Nonselective $\beta$-blockers}

Propranolol is an example of a nonselective $\beta$-blocker with effects on $\beta_{1}$ and $\beta_{2}$ receptors [68]. $\beta_{1}$-Blocking causes a decrease in heart rate. This effect makes $\beta$-blockers (selective or nonselective) an indication for AF according to American Heart Association guidelines [69]. In the landmark AFFIRM trial, $\beta$-blockers were the most effective medications for reducing heart rate, with $70 \%$ of patients achieving the target heart rate [70]. Blocking $\beta_{2}$ causes inhibition of the RAS, lipolysis, glycogenolysis, gluconeogenesis, noradrenaline release and some immune system modulation [68]. The above effects target pathways of ACEI, dyslipidemia and dysglycemia; hence, nonselective $\beta$-blockers may have potential against NAFLD. Indeed, propranolol has shown efficacy in a NAFLD rat model with improvement of serum triglycerides, TNF- $\alpha$, transaminase, insulin resistance and liver histology $(\mathrm{P}<0.05)$ [71]. Further human trials are warranted. 
Lipid-lowering medications

\section{Silymarin}

Silymarin is a flavonoid extract from milk thistle seeds that exhibits antioxidant and anti-inflammatory properties [72]. Silymarin has shown beneficial anthropometric, biochemical, and histologic effects in NAFLD patients [73]. In a trial involving 102 patients undergoing cardiac surgery, silymarin was shown to decrease postoperative proinflammatory cytokines (IL-6 and TNF- $\alpha$ ) [74]. Logistic regression analysis by Ishida et al of 39 patients undergoing cardiac surgery showed that elevated IL- 6 after cardiac surgery was associated with a higher rate of $\mathrm{AF}$ (highest quartile with OR 7.63, 95\%CI 1.06-54.9; $\mathrm{P}=0.04$ ) [75]. Therefore, it is worthwhile to further investigate the role of this medication in AF.

\section{Statins}

Georgescu et al conducted an RCT that included 48 NAFLD patients receiving atorvastatin, losartan, pentoxifylline and ursodeoxycholic acid (UDCA). All groups showed improvement in transaminase and $\gamma$-glutamyl transferase (GGT) [76].

Statins are a group of medications effective against metabolic syndrome and have shown activity against NAFLD and AF. Statins are widely used and accepted in patients with NAFLD [77]. Statins improve NAFLD via various mechanisms. They increase adiponectin, which leads to an improvement in both AF and NAFLD [78]. Statins reduce TGF- $\beta$, improve peroxisomal $\beta$-oxidation and promote nitric oxide synthase [30]. This leads to an antiinflammatory, anti-apoptotic, and anti-oxidative milieu that ameliorates fat accumulation and inflammation in the liver, according to a clinical trial involving $43 \mathrm{NASH}$ patients [79]. A trial with 105 patients, confirmed by a meta-analysis of 13 studies, showed that statins improve AF through reducing oxidative stress and inflammatory markers such as CRP and TNF- $\alpha[80]$.

\section{Obeticholic acid}

Farnesoid X receptor (FXR) is a receptor in the liver that regulates cholesterol metabolism [81]. Obeticholic acid is an FXR agonist that has shown promise in NAFLD in a multicenter US clinical trial involving $141 \mathrm{NASH}$ patients [82]. Animal studies have shown that FXR activation by obeticholic acid leads to a healthier lipid profile by reducing serum low-density lipoprotein (LDL) levels [83]. However, in humans this compound increases LDL and lowers highdensity lipoprotein levels [84]. This may discourage its use in cardiovascular purposes; however, there is insufficient evidence to prove any cardiovascular benefits and/or drawbacks.

\section{Ezetimibe}

Ezetimibe is a drug that binds to the intestinal cholesterol transporter and inhibits cholesterol absorption [85]. According to a consensus panel in the United Kingdom, the addition of ezetimibe to statins reduces LDL by an additional $20 \%$ compared to statins alone [86]. In a meta-analysis of 5 trials involving a total of 199 NAFLD patients, NAFLD activity score was decreased; however, steatosis was not alleviated with ezetimibe [87]. In the landmark IMPROVE-IT trial, which included 18,000 patients with acute coronary syndrome, ezetimibe was associated with an improvement in cardiovascular profile when it was added to statins [88]. In the Simvastatin Ezetimibe in Aortic Stenosis (SEAS) trial, which followed 1421 patients with aortic stenosis for 4.3 years, a combination of simvastatin and ezetimibe was not associated with a lower incidence of AF [89]. Moreover, although lipid-lowering effects were observed, the Ezetimibe and Simvastatin in Hypercholesterolemia Enhances Atherosclerosis Regression (ENHANCE) RCT with 720 familial hypercholesterolemia patients, failed to show an improvement in carotid artery intima-media thickness (a surrogate of atherosclerosis) in familial hypercholesterolemia patients [90].

\section{UDCA}

UDCA is a hydrophilic bile acid that stimulates the excretion of other bile acids by liver cells [91]. Long-term UDCA has shown hepatoprotection in NASH patients [92]. After 12 months of UDCA therapy, in a pilot study involving $40 \mathrm{NASH}$ patients, not only was there an improvement in liver enzymes, but there was also resolution of hepatic steatosis [93]. UDCA showed a benefit in lowering inflammatory markers in 17 male chronic heart failure patients in a single-center RCT [94]. Regarding arrhythmia and AF, in vitro studies have shown a reduction of arrhythmia in cardiomyocytes [95]. However, the efficacy has not been assessed in vivo.

\section{Orlistat}

Orlistat is an over-the-counter weight-loss medication that exerts its effect by inhibiting intestinal lipase [96]. A metaanalysis of 7 clinical trials by Wang et al showed an improvement in transaminase and lipid profile $(\mathrm{P}=0.01)$ in addition to weight loss [97]. In an observational study of 97 NAFLD patients by Khan et al, 4 months' use of orlistat reduced TNF- $\alpha$ in parallel with a reduction in grades of fatty liver [98]. In a multicenter RCT in 33 primary care centers in Sweden, orlistat was shown to lead to not only weight reduction but also a decrease in cardiovascular risks [99]. Orlistat's effects on AF need to be further investigated.

\section{Novel/experimental therapies}

There are novel medications whose effects have not all been fully studied in large multicenter RCTs. However, 
preliminary studies have shown promise in NAFLD and further study is warranted in AF patients, as these medications target common pathways leading to $\mathrm{AF}$ and NAFLD.

\section{Antidiabetic medications}

\section{Elafibranor}

Peroxisome proliferator-activated receptors (PPAR)- $\alpha$ and PPAR- $\delta$ are involved in liver lipid metabolism and insulin sensitivity [100]. Some new medications are designed to utilize these receptors to treat NASH. Elafibranor is a novel agonist of PPAR- $\alpha$ and PPAR- $\delta$ that showed improvements in cardiometabolic parameters, such as lipid profile and insulin sensitivity, in an RCT involving 131 patients [101]. This medication has also been shown to improve hepatic steatosis, liver enzymes, lipid profile and inflammatory markers, such as haptoglobin, in NASH in a multicenter RCT $(\mathrm{P}<0.01)$ [102]. Its effects on cardiovascular mortality and $\mathrm{AF}$ remain to be determined.

\section{Anti-inflammatories and antioxidants}

\section{Quercetin}

Quercetin is a flavonoid present in vegetables and fruits and forms part of our daily diet [103]. Quercetin is an antioxidant and promotes relaxation of cardiovascular smooth muscle, with proven efficacy in reducing atherosclerosis in animal models [104]. Quercetin decreased abdominal obesity, hypertension, dyslipidemia, insulin resistance and hepatic steatosis, and improved cardiovascular remodeling in rats [105]. In animal models, it was found that quercetin exerts its anti-inflammatory and antioxidant activity through its prebiotic effects and by modulating the gut-liver axis [106]. These metabolic and anti-inflammatory effects have already proven beneficial in the cardiovascular system; however, quercetin's effects on AF need to be studied.

\section{Belapectin}

Belapectin is a complex carbohydrate molecule derived from a natural plant that inhibits galectin-3 and exerts antiapoptotic and immunomodulatory effects [107]. In animal models of NASH, the compound decreases hepatic steatosis, proinflammatory cytokines and infiltration, fatty acid oxidation, hepatocyte apoptosis, and fibrosis [108]. In a recent phase $2 \mathrm{~b}$ trial involving patients with cirrhosis secondary to NASH, belapectin showed a clinical effect in reducing portal pressure [109]. At the time of writing this article, clinical cardiovascular effects have not been studied, but we postulate that the medication will have positive effects on AF.

\section{Lipid-lowering medications}

DUR-928

Liver X receptors (LXRs) are involved in liver lipid metabolism [100]. DUR-928 is a natural sulfated oxysterol that inhibits LXR and the inflammatory system, and has shown antisteatotic effects in animal models of NASH [110]. Since the compound decreases inflammation and improves metabolism, further studies in AF are warranted.

\section{Tropifexor/cenicriviroc}

Tropifexor is a non-bile acid FXR agonist that has been shown to reduce steatosis, inflammation and fibrosis in animal models of NASH [111]. Cenicriviroc is an antagonist of C-C Motif Chemokine Receptor types 2 and 5 (CCR2 and CCR5), involved in macrophage activation and the pathogenesis of NASH [112]. Cenicriviroc has shown anti-inflammatory and long-lasting antifibrotic activity in phase $2 \mathrm{~b}$ trials in human subjects who had NASH with fibrosis [113]. The combination of tropifexor and cenicriviroc has shown promise in animal models of NASH, where it led to improvements in inflammation and histology [114]. A phase 2 randomized, double-blind, multicenter trial is underway to determine the efficacy of tropifexor and cenicriviroc in human subjects with NASH [115]. In light of the positive effects on both NASH and inflammatory markers, there is hope for concurrent effects of the combination of tropifexor and cenicriviroc in AF patients.

\section{Aramchol}

Aramchol, a bile acid conjugate that decreases lipid synthesis, has shown promise in NASH [116]. A phase $2 \mathrm{~b}$ randomized clinical trial (ARREST) including 247 patients with NASH showed that aramchol therapy leads to resolution of NASH, a decrease in hepatic steatosis and improvement in glycemic control [116]. The medication was effective in improving metabolic activity in NASH patients; however, its clinical effects on the cardiovascular system need evaluation.

\section{Pradigastat}

Acyl-CoA diacylglycerol acyltransferase (DGAT) is an intestinal enzyme involved in the synthesis of triglycerides, especially the type destined for oxidation [117]. Pradigastat is a DGAT-1 inhibitor that showed promise in a small clinical trial that included 6 patients with familial chylomicronemia syndrome [118]. Studies in NAFLD patients have shown a decrease in liver fat content after 6 months of therapy; however, more than 4 out of 5 patients develop diarrhea due to fat malabsorption [119]. The effect of pradigastat in AF needs to be further investigated. 


\section{Concluding remarks}

There is a multidirectional relationship between NAFLD and AF. The literature suggests that they are linked by proinflammatory, pro-oxidant and atherogenic effects, and a pro-fibrosis environment that stems from and leads to both conditions. There are further mechanisms, such as normal gut flora dysbiosis, effects of the pro-oxidant GGT, and anatomical complications of obesity, that contribute to the aforementioned conditions. Many medications used to treat NAFLD have proven efficacy in AF, as they target dyslipidemia, insulin resistance, the inflammatory system or the RAS. The novel experimental medications for NAFLD have potential for $\mathrm{AF}$, as they target the components of metabolic syndrome or have anti-inflammatory effects. More research is still needed to unveil the pathophysiology, potential mechanisms, and ultimately treatment options targeting both disorders.

\section{References}

1. Chalasani N, Younossi Z, Lavine JE, et al. The diagnosis and management of nonalcoholic fatty liver disease: Practice guidance from the American Association for the Study of Liver Diseases. Hepatology 2018;67:328-357.

2. Younossi ZM, Stepanova M, Afendy M, et al. Changes in the prevalence of the most common causes of chronic liver diseases in the United States from 1988 to 2008. Clin Gastroenterol Hepatol 2011;9:524-530.

3. Lazo M, Hernaez R, Eberhardt MS, et al. Prevalence of nonalcoholic fatty liver disease in the United States: the Third National Health and Nutrition Examination Survey, 1988-1994. Am J Epidemiol 2013;178:38-45.

4. Lindenmeyer CC, McCullough AJ. The natural history of nonalcoholic fatty liver disease-an evolving view. Clin Liver Dis 2018;22:11-21.

5. Younossi ZM, Blissett D, Blissett R, et al. The economic and clinical burden of nonalcoholic fatty liver disease in the United States and Europe. Hepatology 2016;64:1577-1586.

6. Kim EJ, Yin X, Fontes JD, et al. Atrial fibrillation without comorbidities: prevalence, incidence and prognosis (from the Framingham Heart Study). Am Heart J 2016;177:138-144.

7. Naccarelli GV, Varker H, Lin J, Schulman KL. Increasing prevalence of atrial fibrillation and flutter in the United States. Am J Cardiol 2009;104:1534-1539.

8. Grundy SM, Cleeman JI, Daniels SR, et al; National Heart, Lung, and Blood Institute. Diagnosis and management of the metabolic syndrome: an American Heart Association/National Heart, Lung, and Blood Institute scientific statement. Circulation 2005; 112:2735-2752.

9. Wasmer K, Breithardt G, Eckardt L. The young patient with asymptomatic atrial fibrillation: what is the evidence to leave the arrhythmia untreated? Eur Heart J 2014;35:1439-1447.

10. Kodama S, Saito K, Tanaka S, et al. Alcohol consumption and risk of atrial fibrillation: a meta-analysis. J Am Coll Cardiol 2011;57:427-436.

11. Käräjämäki AJ, Hukkanen J, Ukkola $\mathrm{O}$. The association of nonalcoholic fatty liver disease and atrial fibrillation: a review. Ann Med 2018;50:371-380.

12. Mantovani A, Dauriz M, Sandri D, et al. Association between non-alcoholic fatty liver disease and risk of atrial fibrillation in adult individuals: An updated meta-analysis. Liver Int 2019;39:758-769.

13. Sinner MF, Wang N, Fox CS, et al. Relation of circulating liver transaminase concentrations to risk of new-onset atrial fibrillation. Am J Cardiol 2013;111:219-224.

14. Clark JM, Brancati FL, Diehl AM. The prevalence and etiology of elevated aminotransferase levels in the United States. Am J Gastroenterol 2003;98:960-967.

15. Zhang Y, Li P, Miao M, et al. Nonalcoholic fatty liver disease is associated with increased atrial fibrillation risk in an elderly Chinese population: a cross-sectional study. Biomed Res Int 2018;2018:5628749.

16. You SC, Yang P-S, Kim T-H, et al. Non-alcoholic fatty liver disease is independently associated with new onset atrial fibrillation: a nationwide cohort study in Korea. J Am Coll Cardiol 2016;67:854.

17. Käräjämäki AJ, Pätsi OP, Savolainen M, Kesäniemi YA, Huikuri H, Ukkola O. Non-alcoholic fatty liver disease as a predictor of atrial fibrillation in middle-aged population (OPERA Study). PLoS One 2015;10:e142937.

18. Käräjämäki AJ, Kettunen O, Lepojärvi S, et al. Presence of atrial fibrillation is associated with liver stiffness in an elderly Finnish population. PLoS One 2017;12:e173855.

19. Mahfouz RA, Gouda M, Galal I, Ghareb MS. Interatrial septal fat thickness and left atrial stiffness are mechanistic links between nonalcoholic fatty liver disease and incident atrial fibrillation. Echocardiography 2019;36:249-256.

20. Degertekin B, Ozenirler S, Elbeg S, Akyol G. The serum endothelin-1 level in steatosis and NASH, and its relation with severity of liver fibrosis. Dig Dis Sci 2007;52:2622-2628.

21. Abdel Rahman H, Hassan AKM, Abosetta AHS, Kishk YT. Increased left atrial stiffness in patients with atrial fibrillation detected by left atrial speckle tracking echocardiography. Egypt Heart J 2015;67:217-223.

22. Al-Rawahi M, Proietti R, Thanassoulis G. Pericardial fat and atrial fibrillation: Epidemiology, mechanisms and interventions. Int J Cardiol 2015;195:98-103.

23. Ndrepepa G. Myeloperoxidase - a bridge linking inflammation and oxidative stress with cardiovascular disease. Clin Chim Acta 2019;493:36-51.

24. Ellulu MS, Patimah I, Khazaai H, Rahmat A, Abed Y. Obesity and inflammation: the linking mechanism and the complications. Arch Med Sci 2017;13:851-863.

25. Marra F, Lotersztajn S. Pathophysiology of NASH: perspectives for a targeted treatment. Curr Pharm Des 2013;19:5250-5269.

26. Byrne CD, Targher G. NAFLD: a multisystem disease. J Hepatol 2015;62:S47-S64.

27. Ozveren O, Izgi C, Eroglu E, et al. Doppler tissue evaluation of atrial conduction properties in patients with non-alcoholic fattyliver disease. Ultrason Imaging 2016;38:225-235.

28. Targher G, Day CP, Bonora E. Risk of cardiovascular disease in patients with nonalcoholic fatty liver disease. $N$ Engl J Med 2010;363:1341-1350.

29. Mantovani A, Ballestri S, Lonardo A, Targher G. Cardiovascular disease and myocardial abnormalities in nonalcoholic fatty liver disease. Dig Dis Sci 2016;61:1246-1267.

30. Ding YH, Ma Y, Qian LY, et al. Linking atrial fibrillation with non-alcoholic fatty liver disease: potential common therapeutic targets. Oncotarget 2017;8:60673-60683.

31. Warner FJ, Lubel JS, McCaughan GW, Angus PW. Liver fibrosis: a balance of ACEs? Clin Sci (Lond) 2007;113:109-118.

32. Yamamoto E, Dong Y-F, Kataoka K, et al. Olmesartan prevents cardiovascular injury and hepatic steatosis in obesity and diabetes, accompanied by apoptosis signal regulating kinase- 1 inhibition. Hypertension 2008;52:573-580.

33. Olivares-Reyes JA, Arellano-Plancarte A, Castillo-Hernandez JR. 
Angiotensin II and the development of insulin resistance: implications for diabetes. Mol Cell Endocrinol 2009;302:128-139.

34. Lin CS, Pan CH. Regulatory mechanisms of atrial fibrotic remodeling in atrial fibrillation. Cell Mol Life Sci 2008;65:1489-1508.

35. Galea R, Cardillo MT, Caroli A, et al. Inflammation and C-reactive protein in atrial fibrillation: cause or effect? Tex Heart Inst J 2014;41:461-468.

36. Vilar-Gomez E, Martinez-Perez Y, Calzadilla-Bertot L, et al. Weight loss through lifestyle modification significantly reduces features of nonalcoholic steatohepatitis. Gastroenterology 2015;149:367-378.e5.

37. Middeldorp ME, Pathak RK, Meredith M, et al. PREVEntion and regReSsive effect of weight-loss and risk factor modification on atrial fibrillation: the REVERSE-AF study. Europace 2018;20:1929-1935.

38. Pathak RK, Middeldorp ME, Meredith M, et al. Long-term effect of goal-directed weight management in an atrial fibrillation cohort: a long-term follow-up study (LEGACY). J Am Coll Cardiol 2015;65:2159-2169.

39. Khanna D, Baetge C, Simbo S, et al. Effects of diet and exerciseinduced weight loss in sedentary obese women on inflammatory markers, resistin, and visfatin. J Nutr Obes 2017;1:1-13.

40. Askarpour M, Khani D, Sheikhi A, Ghaedi E, Alizadeh S. Effect of bariatric surgery on serum inflammatory factors of obese patients: a systematic review and meta-analysis. Obes Surg 2019;29:2631-2647.

41. Jorge ML, de Oliveira VN, Resende NM, et al. The effects of aerobic, resistance, and combined exercise on metabolic control, inflammatory markers, adipocytokines, and muscle insulin signaling in patients with type 2 diabetes mellitus. Metabolism 2011;60:1244-1252.

42. Colbert LH, Visser M, Simonsick EM, et al. Physical activity, exercise, and inflammatory markers in older adults: findings from the Health, Aging and Body Composition Study. J Am Geriatr Soc 2004;52:1098-1104.

43. Centis E, Marzocchi R, Di Domizio S, Ciaravella MF, Marchesini G. The effect of lifestyle changes in non-alcoholic fatty liver disease. Dig Dis 2010;28:267-273.

44. Li Y, Liu L, Wang B, Wang J, Chen D. Metformin in non-alcoholic fatty liver disease: A systematic review and meta-analysis. Biomed Rep 2013;1:57-64.

45. Chang SH, Wu LS, Chiou MJ, et al. Association of metformin with lower atrial fibrillation risk among patients with type 2 diabetes mellitus: a population-based dynamic cohort and in vitro studies. Cardiovasc Diabetol 2014;13:123.

46. Rena G, Hardie DG, Pearson ER. The mechanisms of action of metformin. Diabetologia 2017;60:1577-1585.

47. Saisho Y. Metformin and inflammation: its potential beyond glucose-lowering effect. Endocr Metab Immune Disord Drug Targets 2015;15:196-205.

48. Boettcher E, Csako G, Pucino F, Wesley R, Loomba R. Metaanalysis: pioglitazone improves liver histology and fibrosis in patients with non-alcoholic steatohepatitis. Aliment Pharmacol Ther 2012;35:66-75.

49. Zhang Z, Zhang X, Korantzopoulos $\mathrm{P}$, et al. Thiazolidinedione use and atrial fibrillation in diabetic patients: a meta-analysis. BMC Cardiovasc Disord 2017;17:96.

50. Miyazaki Y, Mahankali A, Wajcberg E, Bajaj M, Mandarino LJ, DeFronzo RA. Effect of pioglitazone on circulating adipocytokine levels and insulin sensitivity in type 2 diabetic patients. J Clin Endocrinol Metab 2004;89:4312-4319.

51. Poornima I, Brown SB, Bhashyam S, Parikh P, Bolukoglu H, Shannon RP. Chronic glucagon-like peptide-1 infusion sustains left ventricular systolic function and prolongs survival in the spontaneously hypertensive, heart failure-prone rat. Circ Heart Fail 2008;1:153-160.
52. Sokos GG, Nikolaidis LA, Mankad S, Elahi D, Shannon RP. Glucagon-like peptide-1 infusion improves left ventricular ejection fraction and functional status in patients with chronic heart failure. J Card Fail 2006;12:694-699.

53. Nakamura H, Niwano S, Niwano $H$, et al. Liraglutide suppresses atrial electrophysiological changes. Heart Vessels 2019;34:1389-1393.

54. Davies MJ, Bergenstal R, Bode B, et al; NN8022-1922 Study Group. Efficacy of liraglutide for weight loss among patients with type 2 diabetes: the SCALE diabetes randomized clinical trial. JAMA 2015;314:687-699.

55. Blackman A, Foster G, Zammit G, et al. Effect of liraglutide $3.0 \mathrm{mg}$ in individuals with obesity and moderate or severe obstructive sleep apnea: the SCALE Sleep Apnea randomized clinical trial. Int J Obes (Lond) 2016;40:1310-1319.

56. Shiraki A, Oyama J, Komoda H, et al. The glucagon-like peptide 1 analog liraglutide reduces TNF- $\alpha$-induced oxidative stress and inflammation in endothelial cells. Atherosclerosis 2012;221: 375-382.

57. Krasner NM, Ido Y, Ruderman NB, Cacicedo JM. Glucagonlike peptide-1 (GLP-1) analog liraglutide inhibits endothelial cell inflammation through a calcium and AMPK dependent mechanism. PLoS One 2014;9:e97554.

58. Armstrong MJ, Gaunt P, Aithal GP, et al. Liraglutide safety and efficacy in patients with non-alcoholic steatohepatitis (LEAN): a multicentre, double-blind, randomised, placebo-controlled phase 2 study. Lancet 2016;387:679-690.

59. Zhang M, Xu YJ, Mengi SA, Arneja AS, Dhalla NS. Therapeutic potentials of pentoxifylline for treatment of cardiovascular diseases. Exp Clin Cardiol 2004;9:103-111.

60. Du J, Ma YY, Yu CH, Li YM. Effects of pentoxifylline on nonalcoholic fatty liver disease: a meta-analysis. World $J$ Gastroenterol 2014;20:569-577.

61. Chalasani N, Younossi Z, Lavine JE, et al. The diagnosis and management of nonalcoholic fatty liver disease: practice guidance from the American Association for the Study of Liver Diseases. Hepatology 2018;67:328-357.

62. Singh U, Devaraj S, Jialal I. Vitamin E, oxidative stress, and inflammation. Annu Rev Nutr 2005;25:151-174.

63. Rodrigo R, Gutiérrez R, Fernández R, Guzmán P. Ageing improves the antioxidant response against postoperative atrial fibrillation: a randomized controlled trial. Interact Cardiovasc Thorac Surg 2012;15:209-214.

64. Kudo H, Yata Y, Takahara T, et al. Telmisartan attenuates progression of steatohepatitis in mice: role of hepatic macrophage infiltration and effects on adipose tissue. Liver Int 2009;29: 988-996.

65. Shirai Y, Yoshiji H, Noguchi R, et al. Cross talk between tolllike receptor-4 signaling and angiotensin-II in liver fibrosis development in the rat model of non-alcoholic steatohepatitis. $J$ Gastroenterol Hepatol 2013;28:723-730.

66. Qiang G, Zhang L, Yang X, et al. Effect of valsartan on the pathological progression of hepatic fibrosis in rats with type 2 diabetes. Eur J Pharmacol 2012;685:156-164.

67. Ehrlich JR, Hohnloser SH, Nattel S. Role of angiotensin system and effects of its inhibition in atrial fibrillation: clinical and experimental evidence. Eur Heart J 2006;27:512-518.

68. Cruickshank JM. Beta-blockers continue to surprise us. Eur Heart J 2000;21:354-364.

69. Fuster V, Rydén LE, Cannom DS, et al; Heart Rhythm Society. ACC/AHA/ESC 2006 Guidelines for the Management of Patients with Atrial Fibrillation: a report of the American College of Cardiology/American Heart Association Task Force on Practice Guidelines and the European Society of Cardiology Committee for Practice Guidelines (Writing Committee to Revise the 2001 Guidelines for the Management of Patients With Atrial 
Fibrillation): developed in collaboration with the European Heart Rhythm Association and the Heart Rhythm Society. Circulation 2006;114:e257-e354.

70. Olshansky B, Rosenfeld LE, Warner AL, et al; AFFIRM Investigators. The Atrial Fibrillation Follow-up Investigation of Rhythm Management (AFFIRM) study: approaches to control rate in atrial fibrillation. J Am Coll Cardiol 2004;43:1201-1208.

71. Hassankhan B, Yaghmaei P, Parivar K, Ebrahim-Habibi A. Effect of beta-adrenergic blockade in high-fat-induced nonalcoholic fatty liver disease. Arch Pharma Pract 2020;11:87-95.

72. Soto C, Recoba R, Barrón H, Alvarez C, Favari L. Silymarin increases antioxidant enzymes in alloxan-induced diabetes in rat pancreas. Comp Biochem Physiol C Toxicol Pharmacol 2003;136:205-212.

73. Gillessen A, Schmidt HH-J. Silymarin as supportive treatment in liver diseases: a narrative review. $A d v$ Ther 2020;37:1-23.

74. Altaei T. Protective effect of silymarin during coronary artery bypass grafting surgery. Exp Clin Cardiol 2012;17:34-38.

75. Ishida K, Kimura F, Imamaki M, et al. Relation of inflammatory cytokines to atrial fibrillation after off-pump coronary artery bypass grafting. Eur J Cardiothorac Surg 2006;29:501-505.

76. Georgescu EF, Georgescu M. Therapeutic options in nonalcoholic steatohepatitis (NASH). Are all agents alike? Results of a preliminary study. J Gastrointestin Liver Dis 2007;16:39-46.

77. Bays H, Cohen DE, Chalasani N, Harrison SA, The National Lipid Association's Statin Safety Task Force. An assessment by the Statin Liver Safety Task Force: 2014 update. J Clin Lipidol 2014;8:S47-S57.

78. Arnaboldi L, Corsini A. Could changes in adiponectin drive the effect of statins on the risk of new-onset diabetes? The case of pitavastatin. Atheroscler Suppl 2015;16:1-27.

79. Kimura Y, Hyogo H, Yamagishi S, et al. Atorvastatin decreases serum levels of advanced glycation endproducts (AGEs) in nonalcoholic steatohepatitis (NASH) patients with dyslipidemia: clinical usefulness of AGEs as a biomarker for the attenuation of NASH. J Gastroenterol 2010;45:750-757.

80. Wang J, Wang AR, Zhang MJ, Li Y. Effects of atorvastatin on serum high-sensitive C-reactive protein and total cholesterol levels in Asian patients with atrial fibrillation. Am J Ther 2017;24:e20-e29.

81. Lefebvre P, Cariou B, Lien F, Kuipers F, Staels B. Role of bile acids and bile acid receptors in metabolic regulation. Physiol Rev 2009;89:147-191.

82. Neuschwander-Tetri BA, Loomba R, Sanyal AJ, et al; NASH Clinical Research Network. Farnesoid X nuclear receptor ligand obeticholic acid for non-cirrhotic, non-alcoholic steatohepatitis (FLINT): a multicentre, randomised, placebo-controlled trial. Lancet 2015;385:956-965.

83. Singh AB, Dong B, Kraemer FB, Xu Y, Zhang Y, Liu J. Farnesoid $\mathrm{X}$ receptor activation by obeticholic acid elevates liver lowdensity lipoprotein receptor expression by mRNA stabilization and reduces plasma low-density lipoprotein cholesterol in mice. Arterioscler Thromb Vasc Biol 2018;38:2448-2459.

84. Adorini L, Pruzanski M, Shapiro D. Farnesoid X receptor targeting to treat nonalcoholic steatohepatitis. Drug Discov Today 2012;17:988-997.

85. Garcia-Calvo M, Lisnock J, Bull HG, et al. The target of ezetimibe is Niemann-Pick C1-Like 1 (NPC1L1). Proc Natl Acad Sci 2005;102:8132-8137.

86. Mikhailidis DP, Wierzbicki AS, Daskalopoulou SS, et al. The use of ezetimibe in achieving low density lipoprotein lowering goals in clinical practice: position statement of a United Kingdom consensus panel. Curr Med Res Opin 2005;21:959-969.

87. Lee HY, Jun DW, Kim HJ, et al. Ezetimibe decreased nonalcoholic fatty liver disease activity score but not hepatic steatosis. Korean J
Intern Med 2019;34:296-304.

88. Cannon CP, Giugliano RP, Blazing MA, et al; IMPROVE-IT Investigators. Rationale and design of IMPROVE-IT (IMProved Reduction of Outcomes: Vytorin Efficacy International Trial): comparison of ezetimbe/simvastatin versus simvastatin monotherapy on cardiovascular outcomes in patients with acute coronary syndromes. Am Heart J 2008;156:826-832.

89. Bang CN, Greve AM, Boman K, et al. Effect of lipid lowering on new-onset atrial fibrillation in patients with asymptomatic aortic stenosis: the Simvastatin and Ezetimibe in Aortic Stenosis (SEAS) study. Am Heart J 2012;163:690-696.

90. Kastelein JJ, Akdim F, Stroes ES, et al; ENHANCE Investigators. Simvastatin with or without ezetimibe in familial hypercholesterolemia. N Engl J Med 2008;358:1431-1443.

91. Beuers U. Drug insight: Mechanisms and sites of action of ursodeoxycholic acid in cholestasis. Nat Clin Pract Gastroenterol Hepatol 2006;3:318-328.

92. Ratziu V. Treatment of NASH with ursodeoxycholic acid: pro. Clin Res Hepatol Gastroenterol 2012;36 Suppl 1:S41-S45.

93. Laurin J, Lindor KD, Crippin JS, et al. Ursodeoxycholic acid or clofibrate in the treatment of non-alcohol-induced steatohepatitis: a pilot study. Hepatology 1996;23:1464-1467.

94. von Haehling S, Schefold JC, Jankowska EA, et al. Ursodeoxycholic acid in patients with chronic heart failure: a double-blind, randomized, placebo-controlled, crossover trial. $J$ Am Coll Cardiol 2012;59:585-592.

95. Rainer PP, Primessnig U, Harenkamp S, et al. Bile acids induce arrhythmias in human atrial myocardium--implications for altered serum bile acid composition in patients with atrial fibrillation. Heart 2013;99:1685-1692.

96. Narayanaswami V, Dwoskin LP. Obesity: Current and potential pharmacotherapeutics and targets. Pharmacol Ther 2017;170: 116-147.

97. Wang H, Wang L, Cheng Y, Xia Z, Liao Y, Cao J. Efficacy of orlistat in non-alcoholic fatty liver disease: A systematic review and meta-analysis. Biomed Rep 2018;9:90-96.

98. Ali Khan R, Kapur P, Jain A, Farah F, Bhandari U. Effect of orlistat on periostin, adiponectin, inflammatory markers and ultrasound grades of fatty liver in obese NAFLD patients. Ther Clin Risk Manag 2017;13:139-149.

99. Lindgärde F. The effect of orlistat on body weight and coronary heart disease risk profile in obese patients: the Swedish Multimorbidity Study. J Intern Med 2000;248:245-254.

100. Xu P, Zhai Y, Wang J. The role of PPAR and its cross-talk with CAR and LXR in obesity and atherosclerosis. Int J Mol Sci 2018;19:1260.

101. Cariou B, Zaïr Y, Staels B, Bruckert E. Effects of the new dual PPAR $\alpha / \delta$ agonist GFT505 on lipid and glucose homeostasis in abdominally obese patients with combined dyslipidemia or impaired glucose metabolism. Diabetes Care 2011;34:2008-2014.

102. Sookoian S, Pirola CJ. Elafibranor for the treatment of NAFLD: One pill, two molecular targets and multiple effects in a complex phenotype. Ann Hepatol 2016;15:604-609.

103. Formica JV, Regelson W. Review of the biology of quercetin and related bioflavonoids. Food Chem Toxicol 1995;33:1061-1080.

104. Hayek T, Fuhrman B, Vaya J, et al. Reduced progression of atherosclerosis in apolipoprotein E-deficient mice following consumption of red wine, or its polyphenols quercetin or catechin, is associated with reduced susceptibility of LDL to oxidation and aggregation. Arterioscler Thromb Vasc Biol 1997;17:2744-2752.

105. Panchal SK, Poudyal H, Brown L. Quercetin ameliorates cardiovascular, hepatic, and metabolic changes in diet-induced metabolic syndrome in rats. J Nutr 2012;142:1026-1032.

106. Porras D, Nistal E, Martínez-Flórez S, et al. Protective effect of quercetin on high-fat diet-induced non-alcoholic fatty liver 
disease in mice is mediated by modulating intestinal microbiota imbalance and related gut-liver axis activation. Free Radic Biol Med 2017;102:188-202.

107. Yang RY, Hsu DK, Liu FT. Expression of galectin-3 modulates T-cell growth and apoptosis. Proc Natl Acad Sci U S A 1996;93:6737-6742.

108. Iacobini C, Menini S, Ricci C, et al. Galectin-3 ablation protects mice from diet-induced NASH: a major scavenging role for galectin-3 in liver. J Hepatol 2011;54:975-983.

109. Chalasani N, Abdelmalek MF, Garcia-Tsao G, et al; Belapectin (GR-MD-02) Study Investigators. Effects of belapectin, an inhibitor of galectin-3, in patients with nonalcoholic steatohepatitis with cirrhosis and portal hypertension. Gastroenterology 2020;158:1334-1345.

110. Kim LW, Lin WQ. DUR-928, an endogenous regulatory molecule, exhibits anti-inflammatory and antifibrotic activity in a mouse model of NASH. In: Emerging Trends Conference: Emerging Trends in Non alcoholic Fatty Liver Disease. Washington, DC., 2017.

111. Laffitte B, Hernandez E, Kim Y, et al. LJN452 (tropifexor) attenuates steatohepatitis, inflammation, and fibrosis in dietary mouse models of nonalcoholic steatohepatitis. J Hepatol 2018;68 Suppl 1:S341-S342.

112. Tacke F. Cenicriviroc for the treatment of non-alcoholic steatohepatitis and liver fibrosis. Expert Opin Investig Drugs 2018;27:301-311.

113. Ratziu V, Sanyal A, Harrison SA, et al. Cenicriviroc treatment for adults with nonalcoholic steatohepatitis and fibrosis: final analysis of the phase 2b CENTAUR study. Hepatology 2020 Jan
13 [Online ahead of print]. doi: 10.1002/hep.31108

114. Laffitte B, Vig P, Mueller P, Garrett C, Badman MK, Lefebvre E. Combination treatment of Tropifexor (LJN452) and Cenicriviroc shows additive effects in a diet-induced NASH model. Poster 2052, presented at The Liver Meeting ${ }^{\circledR}$, the American Association for the Study of Liver Diseases, Washington, DC, USA, 2017.

115. Pedrosa M, Seyedkazemi S, Francque S, et al. A randomized, double-blind, multicenter, phase $2 \mathrm{~b}$ study to evaluate the safety and efficacy of a combination of tropifexor and cenicriviroc in patients with nonalcoholic steatohepatitis and liver fibrosis: study design of the TANDEM trial. Contemp Clin Trials 2020;88:105889.

116. Ratziu V, Ladron-De-Guevara L, Safadi R, et al. One-year results of the global phase $2 \mathrm{~b}$ randomized placebo-controlled arrest trial of aramchol, a stearoyl CoA desaturase inhibitor, in patients with NASH. Hepatology 2018;6:1448A-1449A.

117. Li C, Li L, Lian J, et al. Roles of Acyl-CoA:diacylglycerol acyltransferases 1 and 2 in triacylglycerol synthesis and secretion in primary hepatocytes. Arterioscler Thromb Vasc Biol 2015;35:1080-1091.

118. Meyers CD, Tremblay K, Amer A, Chen J, Jiang L, Gaudet D. Effect of the DGAT1 inhibitor pradigastat on triglyceride and apoB48 levels in patients with familial chylomicronemia syndrome. Lipids Health Dis 2015;14:8.

119. Sanyal AJ, Cusi K, Patel S, Wright M, Liu C, Keefe DL. Effect of pradigastat, a diacylglycerol acyltransferase 1 inhibitor, on liver fat content in nonalcoholic fatty liver disease. Hepatology 2015;62 (Suppl):1253A. 